\title{
Stress Analysis of Rotary Vibration of Rigid Friction Pile and Stress General Solution of Central Symmetry Plane Elastic Problem
}

\author{
Fu-yao Zhao, Er-xiang Song, and Jun Yang \\ Department of Civil Engineering, Tsinghua University, Beijing 100084, China \\ Correspondence should be addressed to Jun Yang; junyang@tsinghua.edu.cn
}

Received 2 December 2014; Accepted 10 April 2015

Academic Editor: Hakim Naceur

Copyright (c) $2015 \mathrm{Fu}$-yao Zhao et al. This is an open access article distributed under the Creative Commons Attribution License, which permits unrestricted use, distribution, and reproduction in any medium, provided the original work is properly cited.

\begin{abstract}
The rotary vibration of rigid friction pile can be seen approximately as a central symmetry plane problem in elasticity. The stress general solution of central symmetry plane problem in elasticity can be constructed by technique such as the Laurent expansion of the volume force. This solution has some decoupling, generalized, and convergent properties, and it can be used in stress analysis of the rotary vibration of pile. The analysis results show that the maximum value of displacement will not occur at the edge of the pile and the assumption that pile cross section remains unchanged is no longer applicable, if the value of one dimensionless quantity, reflecting the angular frequency of the rotation, radius, and material properties of the pile, is larger than 1.84. Once the rotary vibration of rigid friction pile happens, the pile may lose its bearing capacity under the comprehensive effect of normal and shear stress of the pile-soil interface and it will be very difficult to recover.
\end{abstract}

\section{Introduction}

The seismic response analysis of pile foundation is an important problem in foundation engineering. There is much research about the analysis of situation that pile foundation is under horizontal or vertical dynamic loading. But superstructure will also rotate under seismic action and that will have a great influence on pile foundation which is under abutments or slender transmission towers. For end bearing pile, since the bottom of the pile is fixed, the rotation of the superstructure will cause torsional vibrations of the pile; for rigid friction pile, such as concrete pipe pile, since the modulus of soil is far less than the modulus of the pile (the difference is about 3 orders of magnitude), the rotation of the superstructure will cause rotary vibrations of the whole pile.

For static torsion problem of a single pile, the solution has been found in principle since Saint-Venant problem in elastic mechanics had been solved [1]. There is also much valuable research even considering the effect of soil surrounding pile. Poulos [2] studied the static characteristics of elastic circular pile in Gibson foundation and homogeneous soil under torsional loading with finite difference method. Randolph [3] obtained the analytic expression of torsional stiffness of elastic circular pile in homogeneous soil and soil with its shear modulus changing with depth in the situation ignoring the stress in the soil surrounding the pile. Guo and Randolph [4] present analytical and numerical solutions for the torsional response of piles embedded in nonhomogeneous soil, where the shear modulus profile follows a simple power law with depth.

By comparison, the vibration problem of pile foundation under dynamic torsion load is hard to get analytical solution like static problem because of its complexity, but there is also some valuable research work. Novak and Sachs [5] applied plane strain soil model to study the vibration characteristics of embedded rigid foundation under dynamic torsional loading and derived an approximate analytical solution for torsional vibrations of footings partially embedded in a semiinfinite medium or a stratum. Rajapakse et al. $[6,7]$ studied the dynamic response of a long cylindrical elastic bar which is partially embedded in a homogeneous elastic half-space and is subjected to a harmonic lateral load or to a moment. Cai et al. [8] studied the response of a cylindrical elastic pile embedded in a homogeneous poroelastic medium and 
subjected to torsional loading. Wang et al. [9] developed an analytical solution to investigate the torsional vibration of an end bearing pile embedded in a homogeneous poroelastic medium and subjected to a time-harmonic torsional loading. Zhang [10] derived dynamic governing equations of transversely isotropic saturated soil in cylindrical coordinates on the basis of the dynamic equations of saturated soil and the stress-strain relationships of transversely isotropic medium.

It can be seen from the previous research that many scholars did a lot of work deeply in this field. But, on the one hand, the main objects of this previous research are the dynamic stiffness of pile and displacement response under dynamic loading, and the analysis of stress is rarely mentioned; on the other hand, the previous research is mainly about dynamic torsions of end bearing pile, and the study of the dynamic rotary problem of friction pile is very few. Therefore, this paper will try to analyze this problem. The main research is to carry on theoretical analysis by using analytical methods and to try to develop an approximate analytic solution of the stress distribution of central symmetry plane problem in elasticity. This solution can be used in calculating the stress distribution of friction pile which is under the rotary vibration, and then some advice about antiearthquake analysis will be given.

\section{Stress General Solution of Central Symmetry Plane Problem in Elasticity}

The rotary vibration of a pile can be taken as a particular case of central symmetry plane problem in elasticity. In this section, how to get the stress general solution for such a problem is discussed.

The general solution theory is an important part in Mechanics of Elasticity, and there are some well known results such as Boussinesq-Галёкин [11] general solution, Папкович-Neuber general solution [12], and Тер Мкртичъян-Naghdi-Hsu general solution [13]. Wang has discussed the problems about these solutions' completeness and so on [14]. There is also some valuable research for plane problem, but it is a pity that a lot of classical solutions have adopted the assumption of ignoring the volume force in plane problem under polar coordinates [15]. Although the Kelvin particular solution [16] in general solution theory of elastic mechanics guarantees the possibility of solving elastic mechanics problem including volume force in principle, it is not convenient for use since the singular integral needs to be considered in the calculation because Newton potential has been used in the process of constructing Kelvin particular solution. The Векуа-Мусхелишвили plane general solution [17], which was derived by using Папкович-Neuber general solution, has a similar problem. This section will discuss this problem with stress as unknown and try to get an analytic stress solution of central symmetry plane problem which can be applied easily in elasticity.

The definition of central symmetry plane problem in this paper is given here. After taking the pole of the polar coordinates as the center and rotating the plane by any angle, if the mechanical model can be superposition perfectly with the original one, this problem can be called a central

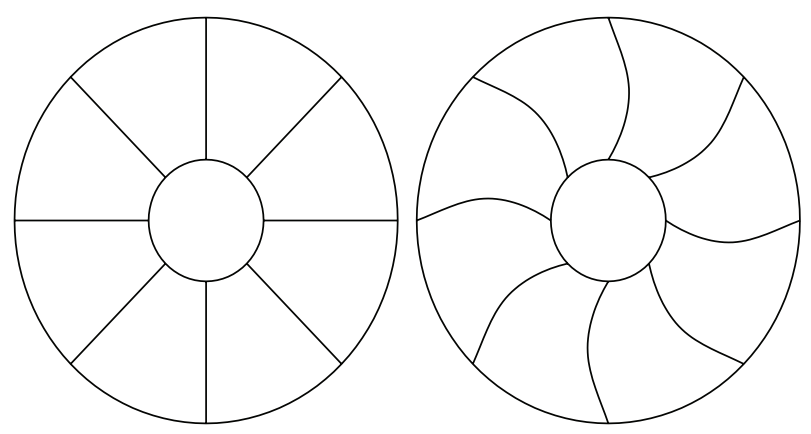

FIgURE 1: Axial symmetry and central symmetry.

symmetry plane problem. In mathematics, it means that all the physical quantities are independent of $\theta$. The difference between central symmetry and axial symmetry in elastic mechanics, specifically speaking, is that the volume force $K_{\theta}$ and the displacement $u_{\theta}$ can be nonzero in central symmetry problem, and so does shear stress $\tau_{r \theta}=\tau_{\theta r}$. Axial symmetry problem can be regarded as a particular case of central symmetry problem (Figure 1).

For central symmetry problem, all the physical quantities are only related to the radius, so $K_{r}=K_{r}(r), K_{\theta}=K_{\theta}(r)$, and the partial derivative with respect to $\theta$ of all the functions will be equal to zero. So, the equilibrium equations and geometric equations in polar coordinates can be, respectively, simplified as

$$
\begin{aligned}
\frac{d \sigma_{r}}{d r}+\frac{\sigma_{r}-\sigma_{\theta}}{r}+K_{r}(r) & =0, \\
\frac{d \tau}{d r}+\frac{2 \tau}{r}+K_{\theta}(r) & =0, \\
\varepsilon_{r} & =\frac{d u_{r}}{d r}, \\
\varepsilon_{\theta} & =\frac{u_{r}}{r}, \\
\gamma & =\frac{d u_{\theta}}{d r}-\frac{u_{\theta}}{r} .
\end{aligned}
$$

Here, $\tau=\tau_{r \theta}=\tau_{\theta r}, \gamma=\gamma_{r \theta}=\gamma_{\theta r}$.

The physical equations remain the same in the case of plane stress problem:

$$
\begin{aligned}
\varepsilon_{r} & =\frac{\sigma_{r}-\mu \sigma_{\theta}}{E}, \\
\varepsilon_{\theta} & =\frac{\sigma_{\theta}-\mu \sigma_{r}}{E}, \\
\gamma & =\frac{\tau}{G}=\frac{2(1+\mu)}{E} \tau .
\end{aligned}
$$

The stress compatibility equation is

$$
\left(\frac{d^{2}}{d r^{2}}+\frac{1}{r} \frac{d}{d r}\right)\left(\sigma_{r}+\sigma_{\theta}\right)=-(1+\mu) \frac{1}{r} \frac{d\left(r K_{r}\right)}{d r} .
$$

For plane strain problem, it just needs to change $E$ to $E /\left(1-\mu^{2}\right)$ and $\mu$ to $\mu /(1-\mu)$. For homogeneous problem 
where volume force $K_{r}=K_{\theta}=0$, central symmetry changes to axial symmetry, and the general solution to the homogeneous form of (1) is

$$
\begin{aligned}
\sigma_{r} & =D_{1}+\frac{D_{2}}{r^{2}}, \\
\sigma_{\theta} & =D_{1}-\frac{D_{2}}{r^{2}}, \\
\tau & =\frac{D_{3}}{r^{2}} .
\end{aligned}
$$

$D_{1}, D_{2}$, and $D_{3}$ are arbitrary constants and can be determined by the boundary condition.

Obviously, the general solution of (1) should be (5) plus a particular solution for the same equations, and the key point is to get the particular solution.

Change the first equation of (1) to

$$
\sigma_{\theta}=\frac{d\left(r \sigma_{r}\right)}{d r}+r K_{r}
$$

For plane stress problem, substitute the former equation into (4) and let

$$
f(r)=\frac{1}{r} \cdot \frac{d^{2}\left[r K_{r}(r)\right]}{d r^{2}}+\frac{2+\mu}{r^{2}} \frac{d\left[r K_{r}(r)\right]}{d r}
$$

and finally we can get

$$
\frac{d^{3} \sigma_{r}}{d r^{3}}+\frac{5}{r} \cdot \frac{d^{2} \sigma_{r}}{d r^{2}}+\frac{3}{r^{2}} \cdot \frac{d \sigma_{r}}{d r}+f(r)=0 .
$$

The former equations are inhomogeneous, and the authors give a particular solution here directly by using variable upper limit integral and a construction method:

$$
\tilde{\sigma}_{r}=\int_{b}^{r} \int_{a}^{t} \frac{\left(s^{2}-t^{2}\right) s^{2}}{2 t^{3}} f(s) d s d t
$$

The appropriate choice of $a, b$ in the above equation can guarantee that the integration process will not have singularity. Considering that (5) is the general solution of the corresponding homogeneous equation, the final normal stress solutions are as follows:

$$
\begin{aligned}
& \sigma_{r}=\widetilde{\sigma}_{r}, \\
& \sigma_{\theta}=\frac{d\left(r \widetilde{\sigma}_{r}\right)}{d r}+r K_{r} .
\end{aligned}
$$

Considering that the second equation of (1) is a first-order Euler equation, the analytical solution can be got directly as follows:

$$
\tau=-\frac{\int r^{2} K_{\theta}(r) d r}{r^{2}}
$$

Synthesizing (5), (10), and (11) we may get the final general solutions of stress:

$$
\begin{aligned}
\sigma_{r} & =D_{1}+\frac{D_{2}}{r^{2}}+\widetilde{\sigma}_{r}, \\
\sigma_{\theta} & =D_{1}-\frac{D_{2}}{r^{2}}+\frac{d\left(r \widetilde{\sigma}_{r}\right)}{d r}+r K_{r}, \\
\tau & =-\frac{\int r^{2} K_{\theta}(r) d r}{r^{2}} .
\end{aligned}
$$

Here, $\widetilde{\sigma}_{r}$ can be determined by (9) and all the arbitrary constants can be determined by the boundary conditions $\left(D_{3}\right.$ has been absorbed by the indefinite integral).

But it can be seen from (9) that the calculation needs to integrate twice to get the normal stress particular solution. When the form of radial volume force is very simple, the integration might get an analytical result. But if the form of the volume force is very complicated, the integration will be very difficult and has to use numerical calculation. Considering the normal stress needs to integrate twice, this solution can only show the numerical value on the point actually, and lots of numerical integrations are needed for stress value on every point. In order to get a solution which is much easier to be applied and enhance the calculation efficiency, we now derive a series solution.

Since all the physical quantities are only dependent on radius $r$, one may expand $K_{r}$ and $K_{\theta}$ as Laurent Series in complex number field according to the power of $r$ and assume that $\sigma_{r}, \sigma_{\theta}$, and $\tau$ have the following form:

$$
\begin{aligned}
K_{r}= & \sum_{\substack{n=-\infty \\
n \neq-1,-3}}^{+\infty} K_{r}^{(n)} r^{n}+K_{r}^{(-1)} r^{-1}+K_{r}^{(-3)} r^{-3}, \\
K_{\theta}= & \sum_{\substack{n=-\infty \\
n \neq-3}}^{+\infty} K_{\theta}^{(n)} r^{n}+K_{\theta}^{(-3)} r^{-3} \\
\widetilde{\sigma}_{r}= & \sum_{\substack{n=-\infty \\
n \neq 0,-2}}^{+\infty} A^{(n)} r^{n}+A^{(0)} \ln r+A^{(-2)} \ln r, \\
\tilde{\sigma}_{\theta}= & \sum_{\substack{n=-\infty \\
n \neq 0,-2}}^{+\infty} B^{(n)} r^{n}+\left(A^{(0)} \ln r+B^{(0)}\right) \\
& +\left(\frac{-A^{(-2)} \ln r}{r^{2}}+\frac{B^{(-2)}}{r^{2}}\right), \\
\tilde{\tau}= & \sum_{\substack{n=-\infty \\
n \neq-2}}^{+\infty} C^{(n)} r^{n}+\frac{C^{(-2)} \ln r}{r^{2}} .
\end{aligned}
$$

The coefficients in the volume force expansion here can be calculated by Cauchy integration in complex number field:

$$
\begin{aligned}
& K_{r}^{(n)}=\frac{1}{2 \pi i} \int_{\Gamma} \frac{K_{r}(\zeta)}{\zeta^{n+1}} d \zeta, \\
& K_{\theta}^{(n)}=\frac{1}{2 \pi i} \int_{\Gamma} \frac{K_{\vartheta}(\zeta)}{\zeta^{n+1}} d \zeta,
\end{aligned}
$$


where $\Gamma$ is a round contour whose center is on the pole and included in the calculation field. All the coefficients in the stress expansion are undetermined.

Substituting the first formula into (7) and (9) successively, the normal stress particular solution of the form as (10) can be obtained. Comparing this particular solution with the third and fourth formulas in (13) we have

$$
\begin{aligned}
& A^{(n)}=-\frac{\mu+n+1}{n(n+2)} K_{r}^{(n-1)}, \\
& B^{(n)}=-\frac{\mu(n+1)+1}{n(n+2)} K_{r}^{(n-1)} .
\end{aligned}
$$

Here, $n \neq 0,-2$, and

$$
\begin{aligned}
A^{(0)} & =-\frac{1+\mu}{2} K_{r}^{(-1)}, \\
B^{(0)} & =\frac{1-\mu}{2} K_{r}^{(-1)}, \\
A^{(-2)} & =\frac{\mu-1}{2} K_{r}^{(-3)}, \\
B^{(-2)} & =\frac{\mu+1}{2} K_{r}^{(-3)} .
\end{aligned}
$$

Substituting $K_{\theta}$ and $\tau$ in (13) into the second formula of (1), we have

$$
\begin{gathered}
\sum_{\substack{n=-\infty \\
n \neq-2}}^{+\infty}\left[(n+2) C^{(n)}+K_{\theta}^{(n-1)}\right] r^{n-1} \\
+\left(C^{(-2)}+K_{\theta}^{(-3)}\right) r^{-3}=0 .
\end{gathered}
$$
have

Setting all the coefficients before the power of $r$ to zero we

$$
\begin{aligned}
C^{(n)} & =-\frac{K_{\theta}^{(n-1)}}{n+2}, \quad n \neq-2, \\
C^{(-2)} & =K_{\theta}^{(-3)} .
\end{aligned}
$$

In principle, the second formula of (1) needs to be supplemented with a general solution: $\tau=D_{3} / r^{2}$, where $D_{3}$ is an arbitrary constant. So, the general solution for the shear stress is

$$
\tau=\sum_{\substack{n=-\infty \\ n \neq-2}}^{+\infty}-\frac{K_{\theta}^{(n-1)}}{n+2} r^{n}+\frac{K_{\theta}^{(-3)} \ln r}{r^{2}}+\frac{D_{3}}{r^{2}} .
$$

Synthesizing (13), (15), (16), and (19) and superposing (5), the general solution of the original equations can be found as

$$
\begin{aligned}
\sigma_{r}= & \sum_{\substack{n=-\infty \\
n \neq 0,-2}}^{+\infty}-\frac{\mu+n+1}{n(n+2)} K_{r}^{(n-1)} r^{n}-\frac{1+\mu}{2} K_{r}^{(-1)} \ln r \\
& +\frac{\mu-1}{2} K_{r}^{(-3)} \frac{\ln r}{r^{2}}+D_{1}+\frac{D_{2}}{r^{2}}, \\
\sigma_{\theta}= & \sum_{\substack{n=-\infty \\
n \neq 0,-2}}^{+\infty}-\frac{\mu(n+1)+1}{n(n+2)} K_{r}^{(n-1)} r^{n} \\
& +\left(\frac{1-\mu}{2} K_{r}^{(-1)}-\frac{1+\mu}{2} K_{r}^{(-1)} \ln r\right) \\
& +\frac{K_{r}^{(-3)}}{2}\left[\frac{(1-\mu) \ln r}{r^{2}}+\frac{\mu+1}{r^{2}}\right]+D_{1}-\frac{D_{2}}{r^{2}}, \\
\tau= & \sum_{\substack{n=-\infty \\
n \neq-2}}^{+\infty}-\frac{K_{\theta}^{(n-1)}}{n+2} r^{n}+\frac{K_{\theta}^{(-3)} \ln r}{r^{2}}+\frac{D_{3}}{r^{2}},
\end{aligned}
$$

where $D_{1}, D_{2}$, and $D_{3}$ are arbitrary constants and can be determined by the boundary conditions.

Obviously, in order to use (20), the Laurent expansion of the volume force is needed, and each term in the expansion needs numerical integration for only once. Compared with (9) and under the situation that the times of numerical integration are the same, (20) will give an approximate solution with some leading terms, but (9) can only give some point values. The advantage of (20) is prominent.

Three theorems about (20) will be given here.

Theorem 1. The normal stress is only related to the radial volume force and the shear stress is only related to the tangential volume force in the central symmetry plane elastic problem.

Proof. It only needs to let $D_{1}=D_{2}=D_{3}=0$ in (20).

Theorem 2. If volume force is analytic in the complex domain, then (1) and (4) have solution (20).

Proof. According to the complex function theory, every function which is analytic in an annular domain can be expanded as Laurent expansion, so formula (20) can be derived by (13).

Theorem 3. If the partial sums of $K_{r}(r)$ and $K_{\theta}(r)$ are bounded (may be not convergent), then (20) is convergent.

Proof. Let $D_{1}=D_{2}=D_{3}=0$ in (20); then

$$
\begin{aligned}
\sigma_{r} & =r \sum_{n=0}^{+\infty}-\frac{\mu+n+2}{(n+1)(n+3)} K_{r}^{(n)} r^{n}, \\
\sigma_{\theta} & =r \sum_{n=0}^{+\infty}-\frac{\mu(n+2)+1}{(n+1)(n+3)} K_{r}^{(n)} r^{n}, \\
\tau & =r \sum_{n=0}^{+\infty}-\frac{1}{n+3} K_{\theta}^{(n)} r^{n} .
\end{aligned}
$$


Assume that

$$
\begin{aligned}
& h_{1}(n)=-\frac{(\mu+n+2)}{\left(n^{2}+4 n+3\right)}, \\
& h_{2}(n)=-\frac{(\mu n+2 \mu+1)}{\left(n^{2}+4 n+3\right)}, \\
& h_{3}(n)=-(n+3)^{-1} .
\end{aligned}
$$

When $n \rightarrow+\infty$, there are three formulas about these factors:

$$
\begin{aligned}
& h_{1}(n) \longrightarrow-(n+4)^{-1}, \\
& h_{2}(n) \longrightarrow-\frac{\mu}{(n+4)}, \\
& h_{3}(n) \longrightarrow-(n+3)^{-1} .
\end{aligned}
$$

They are all arrays monotonically decreasing and approaching zero as the limit. According to the Dirichlet discriminance [18], (20) will be convergent if the partial sums of the infinite series in the first two formulas of (13) are bounded.

Notice. Even when the partial sums of $K_{r}(r)$ and $K_{\theta}(r)$ are unbounded, it is possible that formula (20) is still convergent. The following discussion is about the numerical value purely, such as

$$
\begin{aligned}
& K_{r}^{(n)}=(n+4)^{-1}, \\
& K_{\theta}^{(n)}=(n+3)^{-1} .
\end{aligned}
$$

If $r=1$, it is obvious that the partial sums of the two volume force infinite series are not convergent, but the corresponding items in the three stress components are

$$
\begin{array}{r}
-\frac{\mu+n+2}{(n+1)(n+3)} K_{r}^{(n)} \longrightarrow-\frac{1}{(n+4)^{2}}, \\
-\frac{\mu n+2 \mu+1}{(n+1)(n+3)} K_{r}^{(n)} \longrightarrow-\frac{\mu}{(n+4)^{2}}, \\
-\frac{1}{n+3} K_{\theta}^{(n)} \longrightarrow-\frac{1}{(n+3)^{2}} .
\end{array}
$$

These three stress series are convergent obviously.

In addition, there are two points: firstly the strain and displacement components can be calculated by stress components; secondly, (20) can be generalized to the situation that the index of $r$ is not an integer in the volume force expansion. Since there is no principle difficulty in these two points, the discussion will be omitted here.

\section{Stress Analysis of Pile Rotary Vibration}

The rotary of a pile can be seen as a particular case in central symmetry plane problem in elasticity. In this section, it is attempted to get the analytic stress solution for pile rotary vibrations.

As mentioned before, for the pile foundation, the pile under the structure might have torsional vibration under earthquakes because of the uncertainty of the latter. This torsional effect will be strengthened if the superstructure is irregular. For the rigid friction pile, the source of its bearing capacity is the friction of pile-soil interface. Compared with end bearing piles, the constraint at the bottom of a fiction pile is very weak. Since the stiffness of the pile is very large, this paper assumes that the rotation phases of the pile top and the pile bottom are the same. The movement of the pile can be seen as the rotary vibration of the pile axis and this problem can be seen as a plane strain problem from a certain distance at both ends. This problem can also be seen as a central symmetry problem because all the physical quantities in rotary vibration are independent of $\theta$. The radial and tangential acceleration in polar coordinates can be derived according to [19]

$$
\begin{aligned}
& a_{r}=\frac{\partial^{2} u_{r}}{\partial t^{2}}-\frac{1}{r}\left(\frac{\partial u_{\theta}}{\partial t}\right)^{2}, \\
& a_{\theta}=\frac{2}{r} \cdot \frac{\partial u_{r}}{\partial t} \cdot \frac{\partial u_{\theta}}{\partial t}+\frac{\partial^{2} u_{\theta}}{\partial t^{2}} .
\end{aligned}
$$

Considering that $u_{\theta}$ will be given as an initial condition in the calculation in the following, $u_{r}$ is caused by $\sigma_{r}\left(\sigma_{r}\right.$ is related to $u_{\theta}$ because of the centrifugal force), and the materials (concrete, soil) discussed in this paper have high modulus, this paper assumes that $u_{r}$ is far less than $u_{\theta}$. Also, the calculation in the following limits the range of rotary angular frequency $v$ because of some approximation. As a result, we assume that

$$
\begin{aligned}
& K_{r}=-\rho a_{r} \approx \frac{\rho}{r}\left(\frac{\partial u_{\theta}}{\partial t}\right)^{2}, \\
& K_{\theta}=-\rho a_{\theta} \approx-\rho \frac{\partial^{2} u_{\theta}}{\partial t^{2}} .
\end{aligned}
$$

Taking the third formula of (3), the third formula of (2), and the second formula of (27) into the second formula of (1), we have

$$
\frac{\partial^{2} u_{\theta}}{\partial r^{2}}+\frac{1}{r} \frac{\partial u_{\theta}}{\partial r}-\frac{u_{\theta}}{r^{2}}=\frac{1}{c_{s}^{2}} \frac{\partial^{2} u_{\theta}}{\partial t^{2}}
$$

where $c_{s}=\sqrt{G / \rho}$.

Assume that $u_{\theta}=R(r) T(t)$, and by using the method of separation of variables to solve (28) we can get

$$
\begin{aligned}
& T(t)=C_{1} \sin (v t)+C_{2} \cos (v t), \\
& R(r)=C_{3} \frac{c_{s}}{v} J_{1}\left(\frac{v}{c_{s}} r\right)+C_{4} \frac{c_{s}}{v} Y_{1}\left(\frac{v}{c_{s}} r\right),
\end{aligned}
$$

where $J_{1}$ and $Y_{1}$ are the Bessel functions of the first and second kinds of the first order and $v$ is the rotary angular frequency. $C_{1}, C_{2}, C_{3}$, and $C_{4}$ are all arbitrary constants. Assume that 


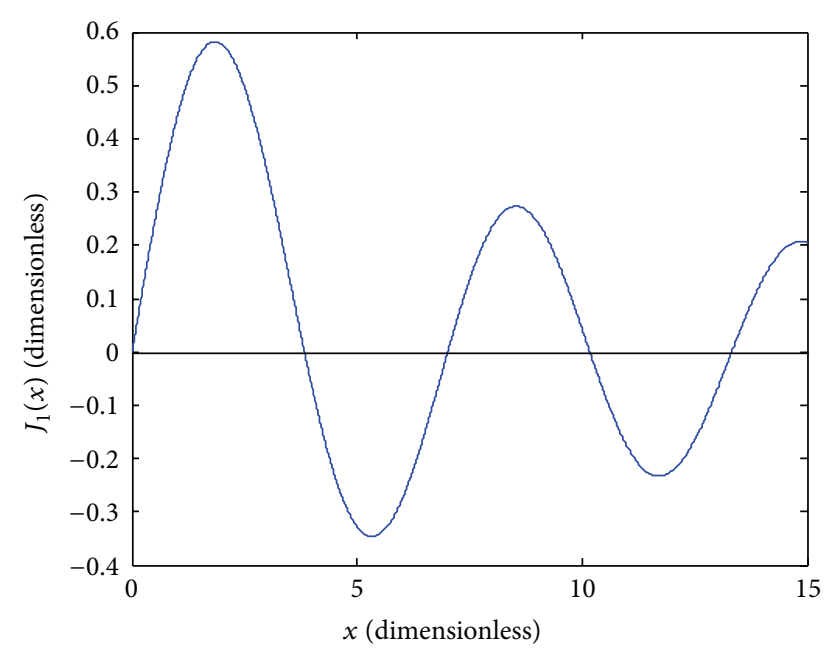

FIGURE 2: The image of $J_{1}(x)$.

$\left.u_{\theta}\right|_{t=0}=0$, so $C_{2}=0$. Since the two parts about the radius in the displacement component have arbitrary constants, we can assume that $C_{1}=1$. So,

$$
u_{\theta}=\left[C_{3} \frac{c_{s}}{v} J_{1}\left(\frac{v}{c_{s}} r\right)+C_{4} \frac{c_{s}}{v} Y_{1}\left(\frac{v}{c_{s}} r\right)\right] \sin (v t) .
$$

Considering the most general form, the solution of displacement should be the sum of $v=v_{1}, v_{2}, \ldots, v_{n}, \ldots$ In order to simplify the analysis process, this paper only uses (30) as the solution of the displacement, which means that the rotary vibration of the pile has only one certain frequency. We will use $p$ on behalf of pile and $s$ on behalf of soil in the following. Assume that the radius of the pile is $r_{0}$ (here it considers solid pile), and we have $\left.u_{\theta p}\right|_{r=0}=0$ in the center of the circle. When $r \rightarrow 0$, we have $Y_{1}(r) \rightarrow \infty$, so $C_{4}=0$. Let $C_{3}=A$ and obviously it has the meaning of something like amplitude ( $A$ will be called similar-amplitude in the following pages). The tangential displacement of the pile is

$$
u_{\theta p}=A \frac{c_{s p}}{v} J_{1}\left(\frac{v}{c_{s p}} r\right) \sin (v t) .
$$

That means, although the radius of the pile under stationary state is a straight linear segment originally, it will become curved because of the effect of inertial force in the rotary problem (Figure 2). But the independent variables which make $J_{1}$ get the first maximal value are 1.84; it means that the tangential displacement $u_{\theta p}$ at the cross section of the pile still increases with the increase of the radius when the dimensionless independent variables of $J_{1}, x=v r / c_{s p}$, do not exceed 1.84. So, the radius can be seen as a linear segment approximately under the situation that the angle of rotation is not large (the corresponding physical quantity is $A$ which has a similar physical significance) and the demand of accuracy is not much. The general precast concrete pile usually has a high $c_{s p}$ (more than $2000 \mathrm{~m} / \mathrm{s}$ ). If we make $r=0.5 \mathrm{~m}$ and $x=1.84$, the corresponding $v$ will be about $7360 \mathrm{~Hz}$. So, in the rotary vibration of the whole pile, the deformation of the radius can be ignored when the numerical value of $v$ is in the range of $0 \sim 7360 \mathrm{~Hz}$. But if the actual numerical values of the physical quantities lead to the fact that the value of $x$ is more than 1.84 , the rotary vibration of the pile can no longer be seen as rigid rotation because the maximal value of tangential displacement is inside the pile cross section, not in the outer edge.

In order to calculate the volume inertial force in the pile rotary problem, substitute (31) into (27):

$$
\begin{aligned}
& K_{r p}=\frac{A^{2} G_{p} \cos ^{2}(v t)}{r} J_{1}^{2}\left(\frac{v}{c_{s p}} r\right), \\
& K_{\theta p}=A \rho_{p} v c_{s p} \sin (v t) J_{1}\left(\frac{v}{c_{s p}} r\right) .
\end{aligned}
$$

If the stress is calculated with the integral solution of central symmetry plane problem mentioned above, then

$$
\begin{aligned}
f(r)= & \frac{1}{r} \cdot \frac{\partial^{2}\left[r K_{r p}(r)\right]}{\partial r^{2}}+\frac{2+\mu^{\prime}}{r^{2}} \frac{\partial\left[r K_{r p}(r)\right]}{\partial r} \\
= & \frac{A^{2} G_{p} \cos ^{2}(v t)}{r} \cdot \frac{\partial^{2}\left[J_{1}^{2}\left(\left(v / c_{s p}\right) r\right)\right]}{\partial r^{2}} \\
& +\frac{\left(2+\mu^{\prime}\right) A^{2} G_{p} \cos ^{2}(v t)}{r^{2}} \frac{\partial\left[J_{1}^{2}\left(\left(v / c_{s p}\right) r\right)\right]}{\partial r} .
\end{aligned}
$$

Because this is a plane strain problem, here $\mu^{\prime}=\mu /(1-$ $\mu)$. Obviously if we substitute the former formula into (9) to get a solution, the calculation will be very difficult. So, the series solution derived in this paper will be used here. For this purpose, the astringency of the volume force series solution needs to be considered. Since the radial volume force is expressed by the Bessel functions of the first kind of the first order, its infinite series form is

$$
J_{1}(x)=\sum_{m=0}^{\infty} \frac{(-1)^{m}}{m !(m+1) !}\left(\frac{x}{2}\right)^{2 m+1} .
$$

It is easy to know that this series is absolutely convergent in the whole field of real number. According to the Cauchy theorem [18], some leading terms are taken to calculate $K_{r p}$, $K_{\theta p}$ as follows:

$$
\begin{aligned}
& J_{1}^{2}\left(\frac{v}{c_{s p}} r\right)=\frac{1}{4}\left(\frac{v}{c_{s p}}\right)^{2} r^{2}-\frac{1}{16}\left(\frac{v}{c_{s p}}\right)^{4} r^{4}+\frac{5}{768} \\
& \cdot\left(\frac{v}{c_{s p}}\right)^{6} r^{6}+\cdots, \\
& K_{r p}=A^{2} G_{p} \cos ^{2}(v t)\left[\frac{1}{4}\left(\frac{v}{c_{s p}}\right)^{2} r-\frac{1}{16}\left(\frac{v}{c_{s p}}\right)^{4} r^{3}\right. \\
& \left.+\frac{5}{768} \cdot\left(\frac{v}{c_{s p}}\right)^{6} r^{5}+\cdots\right], \\
& K_{\theta p}=A \rho_{p} v c_{s p} \sin (v t) \sum_{m=0}^{\infty} \frac{(-1)^{m}}{m !(m+1) !}\left(\frac{v r}{2 c_{s p}}\right)^{2 m+1} .
\end{aligned}
$$


Substitute the former result into (20):

$$
\begin{aligned}
& \sigma_{r p}=D_{1}+\frac{D_{2}}{r^{2}}-\frac{\mu^{\prime}+3}{8} A^{2} G_{p} \cos ^{2}(v t)\left(\frac{v r}{2 c_{s p}}\right)^{2} \\
& +\frac{\mu^{\prime}+5}{24} A^{2} G_{p} \cos ^{2}(v t)\left(\frac{v r}{2 c_{s p}}\right)^{4} \\
& -\frac{\mu^{\prime}+7}{48} A^{2} G_{p} \cos ^{2}(v t) \frac{5}{12} \cdot\left(\frac{v r}{2 c_{s p}}\right)^{6} \\
& +\cdots \text {, } \\
& \sigma_{\theta p}=D_{1}-\frac{D_{2}}{r^{2}}-\frac{3 \mu^{\prime}+1}{8} A^{2} G_{p} \cos ^{2}(v t)\left(\frac{v r}{2 c_{s p}}\right)^{2} \\
& +\frac{5 \mu^{\prime}+1}{24} A^{2} G_{p} \cos ^{2}(v t)\left(\frac{v r}{2 c_{s p}}\right)^{4} \\
& -\frac{7 \mu^{\prime}+1}{48} A^{2} G_{p} \cos ^{2}(v t) \frac{5}{12} \cdot\left(\frac{v r}{2 c_{s p}}\right)^{6} \\
& +\cdots, \\
& \tau_{p}=A G_{s p} \sin (v t) \sum_{m=0}^{+\infty} \frac{(-1)^{m+1}}{m !(m+2) !}\left(\frac{v r}{2 c_{s p}}\right)^{2 m+2} \\
& +\frac{D_{3}}{r^{2}} \text {. }
\end{aligned}
$$

Stress solution should have no singularity in the origin: $D_{2}=D_{3}=0$.

If the soil around the pile is not considered, that is, $\left.\sigma_{r}\right|_{r=R}=0$, we can get

$$
\begin{aligned}
D_{1} & \approx A^{2} G_{p} \cos ^{2}(v t)\left[\frac{\mu^{\prime}+3}{8}\left(\frac{v R}{2 c_{s p}}\right)^{2}\right. \\
& \left.-\frac{\mu^{\prime}+5}{24}\left(\frac{v R}{2 c_{s p}}\right)^{4}+\frac{\mu^{\prime}+7}{48} \frac{5}{12} \cdot\left(\frac{v R}{2 c_{s p}}\right)^{6}\right] .
\end{aligned}
$$

Since $\cos ^{2}(v t) \leq 1, \sin (v t) \leq 1$, the maximal value of the stress during the time period is

$$
\begin{gathered}
\sigma_{r p \max (t)} \approx A^{2} G_{p}\left\{\frac{\mu^{\prime}+3}{8}\left[\left(\frac{v R}{2 c_{s p}}\right)^{2}-\left(\frac{v r}{2 c_{s p}}\right)^{2}\right]\right. \\
-\frac{\mu^{\prime}+5}{24}\left[\left(\frac{v R}{2 c_{s p}}\right)^{4}-\left(\frac{v r}{2 c_{s p}}\right)^{4}\right]+\frac{\mu^{\prime}+7}{48} \frac{5}{12} \\
\left..\left[\left(\frac{v R}{2 c_{s p}}\right)^{6}-\left(\frac{v r}{2 c_{s p}}\right)^{6}\right]\right\},
\end{gathered}
$$

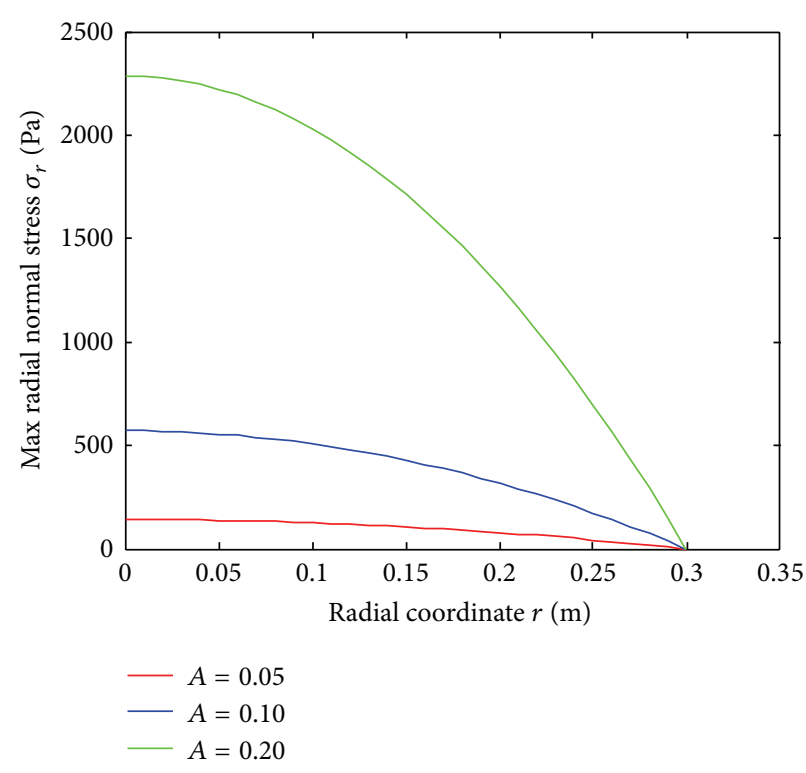

FIGURE 3: Max radial normal stress of pile.

$$
\begin{gathered}
\sigma_{\theta p \max (t)} \approx A^{2} G_{p}\left\{\left[\frac{\mu^{\prime}+3}{8}\left(\frac{v R}{2 c_{s p}}\right)^{2}\right.\right. \\
\left.-\frac{3 \mu^{\prime}+1}{8}\left(\frac{v r}{2 c_{s p}}\right)^{2}\right]-\left[\frac{\mu^{\prime}+5}{24}\left(\frac{v R}{2 c_{s p}}\right)^{4}\right. \\
\left.-\frac{5 \mu^{\prime}+1}{24}\left(\frac{v r}{2 c_{s p}}\right)^{4}\right]+\left[\frac{\mu^{\prime}+7}{48} \frac{5}{12} \cdot\left(\frac{v R}{2 c_{s p}}\right)^{6}\right. \\
\left.\left.-\frac{7 \mu^{\prime}+1}{48} \frac{5}{12} \cdot\left(\frac{v r}{2 c_{s p}}\right)^{6}\right]\right\}, \\
\tau_{p \max (t)}=A G_{s p} \sum_{m=0}^{+\infty} \frac{(-1)^{m+1}}{m !(m+2) !}\left(\frac{v r}{2 c_{s p}}\right)^{2 m+2} .
\end{gathered}
$$

This is the final stress solution.

In the following example, the diameter of the pile is $600 \mathrm{~mm}(r=0.3 \mathrm{~m})$. The material of the pile is C30 concrete, and its shear modulus is about $1.25 \times 10^{10} \mathrm{~Pa}$, its density is $2.5 \times 10^{3} \mathrm{~kg} / \mathrm{m}^{3}$, and Poisson's ratio is 0.2 . Taking circular frequency to be $50 \mathrm{~Hz}$ (the corresponding frequency $f=$ $v /(2 \pi)$ is about $7.96 \mathrm{~Hz})$, we adjust the similar-amplitude $A$ and then calculate the stress. The results are shown in Figures 3 5.

According to Figures 3 5, for the rotary problem of rigid pile, the normal stress is more sensitive to similar-amplitude $A$ than the shear stress. The reason is that, according to (38), the normal stress is proportional to $A^{2}$ and the shear stress is proportional to $A$. Noting that the value of $A$ is very small, the difference of numerical values between the normal stress and the shear stress can reflect the rationality of the assumption in the beginning of this section $\left(u_{r}\right.$ is far less than $\left.u_{\theta}\right)$ to a certain extent. Besides, when the rotary angular frequency is no more 


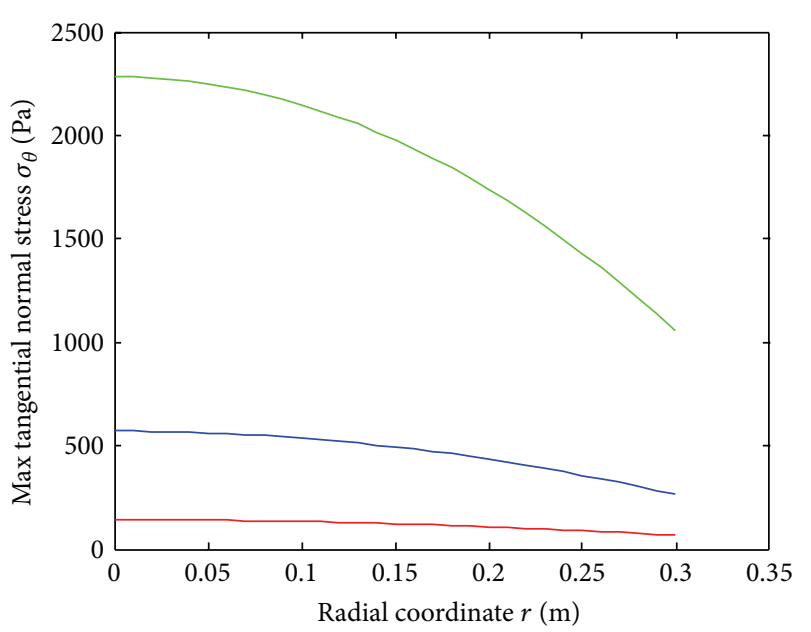

$\begin{aligned} A & =0.05 \\ -A & =0.10 \\ A & =0.20\end{aligned}$

FIGURE 4: Max tangential normal stress of pile.

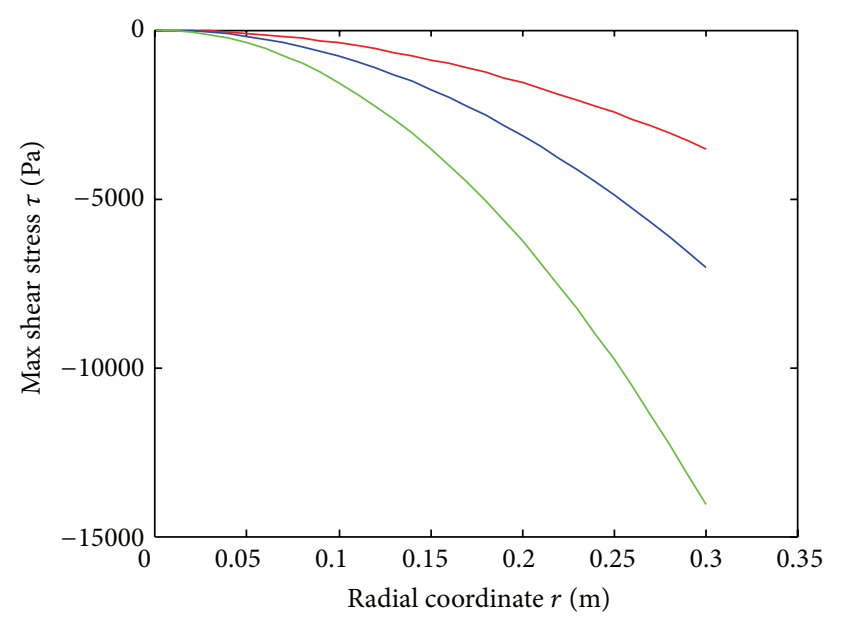

$\begin{aligned} A & =0.05 \\ -A & =0.10 \\ A & =0.20\end{aligned}$

FIgURE 5: Max shear stress of pile.

than $50 \mathrm{~Hz}$, the normal stress of the pile will not exceed $6 \mathrm{kPa}$, and the shear stress is relatively large because the numerical value can be about $15 \mathrm{kPa}$. But the elasticity modulus of C30 concrete is very large (about $30 \mathrm{GPa}$ ), so the deformation of the pile is very small. As mentioned in the previous analysis that the pile radius can be seen as a linear segment approximately, we may safely draw the conclusion that the rotary motion can be seen as the rotations of rigid body. This conclusion is based on the ignoring of the soil around the pile. The modulus difference between soil and concrete is three orders of magnitude, and the order of magnitude of the stress is usually $10^{4} \mathrm{~Pa}$ far from the degree which can make concrete appear obvious deformation. So, in the analysis of the next section, this conclusion will be used as a fundamental assumption, and the rationality of this assumption can be seen in the calculation results in the next section.

For comparison, the traditional torsional vibration theory of pile will be discussed here. Obviously, the existing theory of torsional vibration that takes the pile as a one-dimensional structural element is based on static torsional displacement form [20], and it contains a basic assumption: the radius of the pile cross section maintains linear segment during the torsional vibration. Compared with the previous analysis of rotary problem, it can be seen that this assumption is appropriate only when the vibration frequency is low. But if the vibration frequency is high, the largest tangential displacement does not appear on the surface of the pile because of the effect of the inertial force and the bending of the original radius cannot be ignored any more. In this situation, if we still use the traditional torsional vibration theory, the calculation results might have large error. Although the specific quantitative analysis is very difficult, the rotary vibration solution can be consulted to some extent. If the dimensionless quantity $v r / c_{s p}$ is bigger than 1.84 , the traditional torsional vibration solution is no longer appropriate.

\section{Stress Analysis for Soil around the Pile}

Based on the conclusion above, the displacement of the pile will be given as a Dirichlet boundary condition for the soil around the pile, under the situation that the dimensionless quantity $v r / c_{s p}$ is not larger than 1.84 , in the soil stress analysis in this section.

Obviously, the movement of the soil around the pile meets (28), so its displacement has a form like that shown by (30). Since $J_{1}$ and $Y_{1}$ are both infinite series, if we use the sum of $J_{1}$ and $Y_{1}$ to calculate the Cauchy product of this sum's square, the process will be extremely complex. Noting that the value of that dimensionless quantity $v r / c_{s p}$ is usually very small, the first-order approximate solution of $J_{1}$ and the asymptotic solution of $Y_{1}$ can be written as [21]

$$
\begin{aligned}
& J_{1}(x) \approx \frac{x}{2}, \\
& Y_{1}(x) \approx-\frac{2}{(\pi x)} .
\end{aligned}
$$

So, (30) can be rewritten as

$$
u_{\theta}=\left(\frac{C_{3} r}{2}-\frac{2 C_{4} c_{s}^{2}}{\pi v^{2} r}\right) \sin (v t)
$$

Assume that the radius of the pile is $R$, the soil-pile interface will not be separated, the farthest influence area of rotary vibration of soil around the pile is $r=R_{1}$ (the soil outside $r=R_{1}$ is static), the maximum rotary angle is $\theta$, and the circular frequency is $v$. The boundary conditions are

$$
\begin{aligned}
& \left.u_{\theta}\right|_{r=R}=R \theta \sin (v t), \\
& \left.u_{\theta}\right|_{r=R_{1}}=0,
\end{aligned}
$$




$$
\begin{gathered}
\left.u_{r}\right|_{r=R}=0, \\
\left.u_{r}\right|_{r=R_{1}}=0 .
\end{gathered}
$$

According to the first two formulas of (41), we have

$$
\begin{aligned}
C_{3} & =\frac{2 R \theta \cdot R}{R^{2}-R_{1}^{2}}, \\
C_{4} & =\frac{\pi v^{2} R \theta \cdot R \cdot R_{1}^{2}}{2 c_{s}^{2}\left(R^{2}-R_{1}^{2}\right)} .
\end{aligned}
$$

So,

$$
u_{\theta}=\left(\frac{R^{2} \theta r}{R^{2}-R_{1}^{2}}-\frac{R_{1}^{2} R^{2} \theta}{\left(R^{2}-R_{1}^{2}\right) r}\right) \sin (v t) .
$$

Integrating (27), (30), and (42), we have

$$
\begin{aligned}
& K_{r}=\rho v^{2} \cos ^{2}(v t) \frac{(R \theta)^{2} \cdot R^{2}}{\left(R^{2}-R_{1}^{2}\right)^{2}}\left(r-\frac{2 R_{1}^{2}}{r}+\frac{R_{1}^{4}}{r^{3}}\right), \\
& K_{\theta}=\rho v^{2} \sin (v t) \frac{\theta R^{2}\left(r^{2}-R_{1}^{2}\right)}{\left(R^{2}-R_{1}^{2}\right) r} .
\end{aligned}
$$

If we use the last formula of (38) to calculate the shear stress, the arbitrary constant $D_{3}$ is not easy to determine. Since $u_{\theta}$ has already been calculated, we can use the third formula of (2) and the third formula of (3), (40), and (42) to get

$$
\tau=G\left(\frac{\partial u_{\theta}}{\partial r}-\frac{u_{\theta}}{r}\right)=\frac{2 R_{1}^{2} R^{2} \theta G}{R^{2}-R_{1}^{2}} \cdot \frac{\sin (v t)}{r^{2}}
$$

It can be seen that (43) and (45) will change to the solution of the corresponding static problem if the time term $\sin (v t)$ is taken away (it is a static problem; $K_{r}=K_{\theta}=0$ ). It means that, without the time term $\sin (v t)$, the tangential displacement and the shear stress are very close to the static solution if the value of the dimensionless quantity $x=v r / c_{s p}$ is very small (if radius $r$ and velocity $c_{s p}$ are certain, rotary frequency should be very low).

By contrast, the normal stress calculation formula is more complicated. According to (20), the normal stress in the soil is

$$
\begin{aligned}
& \sigma_{r}=m(r) \cos ^{2}(v t)+D_{1}+\frac{D_{2}}{r^{2}}, \\
& \sigma_{\theta}=n(r) \cos ^{2}(v t)+D_{1}-\frac{D_{2}}{r^{2}},
\end{aligned}
$$

where

$$
\begin{aligned}
& m(r)=\rho v^{2} \frac{(R \theta)^{2} \cdot R^{2}}{\left(R^{2}-R_{1}^{2}\right)^{2}}\left[-\frac{3+\mu^{\prime}}{8} r^{2}+\left(1+\mu^{\prime}\right) R_{1}^{2} \ln r\right. \\
& \left.-\frac{\left(1-\mu^{\prime}\right) \ln r}{2 r^{2}} R_{1}^{4}\right], \\
& n(r)=\rho v^{2} \frac{(R \theta)^{2} \cdot R^{2}}{\left(R^{2}-R_{1}^{2}\right)^{2}}\left\{-\frac{1+3 \mu^{\prime}}{8} r^{2}\right. \\
& +\left[\left(1+\mu^{\prime}\right) \ln r-\left(1-\mu^{\prime}\right)\right] R_{1}^{2} \\
& \left.+\frac{\left(1-\mu^{\prime}\right) \ln r+\left(1+\mu^{\prime}\right)}{2 r^{2}} R_{1}^{4}\right\}
\end{aligned}
$$

where $\mu^{\prime}=\mu /(1-\mu)$, that is, the same as in the third section of this paper. By the second formula of (2) and the third formula of (3), we have $u_{r} / r=\left(\sigma_{\theta}-\mu^{\prime} \sigma_{r}\right) / E^{\prime}$. For the same reason (plane strain problem), here $E^{\prime}=E /\left(1-\mu^{2}\right)$.

Here, the last two formulas of (41) can be rewritten as

$$
\left.\left(\sigma_{\theta}-\mu^{\prime} \sigma_{r}\right)\right|_{r=R}=\left.\left(\sigma_{\theta}-\mu^{\prime} \sigma_{r}\right)\right|_{r=R 1}=0
$$

In order to determine $D_{1}$ and $D_{2}$, substituting (46) into (48), we have

$$
\begin{aligned}
D_{1} & \frac{\mu^{\prime}\left[R^{2} m(R)-R_{1}^{2} m\left(R_{1}\right)\right]-\left[R^{2} n(R)-R_{1}^{2} n\left(R_{1}\right)\right]}{\left(R^{2}-R_{1}^{2}\right)\left(1-\mu^{\prime}\right)} \\
& \cdot \cos ^{2}(v t), \\
D_{2} & =\frac{\mu^{\prime}\left[m(R)-m\left(R_{1}\right)\right]-\left[n(R)-n\left(R_{1}\right)\right]}{1+\mu^{\prime}} \\
& \cdot \frac{R_{1}^{2} R^{2} \cos ^{2}(v t)}{R^{2}-R_{1}^{2}} .
\end{aligned}
$$

Because $\cos ^{2}(v t) \leq 1$, when $t=2 k \pi / v$ ( $k$ is a negative integer), the normal stress distribution in the soil is

$$
\begin{aligned}
& \sigma_{r \max (t)}=m(r) \\
& +\frac{\mu^{\prime}\left[R^{2} m(R)-R_{1}^{2} m\left(R_{1}\right)\right]-\left[R^{2} n(R)-R_{1}^{2} n\left(R_{1}\right)\right]}{\left(R^{2}-R_{1}^{2}\right)\left(1-\mu^{\prime}\right)} \\
& +\frac{\mu^{\prime}\left[m(R)-m\left(R_{1}\right)\right]-\left[n(R)-n\left(R_{1}\right)\right]}{1+\mu^{\prime}} \\
& +\frac{R_{1}^{2} R^{2}}{\left(R^{2}-R_{1}^{2}\right) r^{2}},
\end{aligned}
$$




$$
\begin{aligned}
& \sigma_{\theta \max (t)}=n(r) \\
& +\frac{\mu^{\prime}\left[R^{2} m(R)-R_{1}^{2} m\left(R_{1}\right)\right]-\left[R^{2} n(R)-R_{1}^{2} n\left(R_{1}\right)\right]}{\left(R^{2}-R_{1}^{2}\right)\left(1-\mu^{\prime}\right)} \\
& -\frac{\mu^{\prime}\left[m(R)-m\left(R_{1}\right)\right]-\left[n(R)-n\left(R_{1}\right)\right]}{1+\mu^{\prime}} \\
& \cdot \frac{R_{1}^{2} R^{2}}{\left(R^{2}-R_{1}^{2}\right) r^{2}} .
\end{aligned}
$$

Because $\sin (v t) \leq 1$, when $t=(2 k+0.5) \pi / v(k$ is a negative integer), the shear stress distribution in the soil is

$$
\tau_{s \max (t)}=\frac{2 R_{1}^{2} R^{2} \theta G_{s}}{\left(R^{2}-R_{1}^{2}\right) r^{2}} .
$$

If the scope of our investigation is the whole plane, $R_{1} \rightarrow$ $\infty$, the distribution of $\tau_{\max }$ has a maximal value (the absolute value is minimal value): $\tau_{\max }=-2 R^{2} \theta G_{s} / r^{2}$. Obviously, in the whole plane, the minimal value of the absolute value is at the point $r=R$ and

$$
\left.\tau_{\max }\right|_{r=R}=-2 \theta G_{s}
$$

In the following example, the diameter of the pile is $600 \mathrm{~mm}(r=0.3 \mathrm{~m})$. Assume that the shear modulus of the soil is about $2.5 \times 10^{7} \mathrm{~Pa}$, its density is $2.5 \times 10^{3} \mathrm{~kg} / \mathrm{m}^{3}$, Poisson's ratio is 0.2 , and $R_{1}=2 \mathrm{~m}$. Taking circular frequency to be $50 \mathrm{~Hz}$ (the corresponding frequency $f=v /(2 \pi)$ is about $7.96 \mathrm{~Hz}$ ), we adjust circular frequency $\theta$ and calculate the stress. The results are shown in Figures $6 \sim 8$.

According to Figures $6 \sim 8$, the normal stress is more sensitive to the maximum rotary angle $\theta$ than the shear stress. It is for the same reason as mentioned in the second section; the normal stress is proportional to $\theta^{2}$ and the shear stress is proportional to $\theta$. This difference also indicates that the assumption in the second section $\left(u_{r}\right.$ is far less than $\left.u_{\theta}\right)$ can also be used in this section. The physical meaning of the calculation results will be discussed here.

The normal stress will be analyzed first. It can be seen that $\sigma_{r}$ in the soil-pile interface $(r=0.3 \mathrm{~m})$ is always positive (tensile stress). When the circular frequency $v=50 \mathrm{~Hz}$ (corresponding frequency is about $7.96 \mathrm{~Hz}$ ) and $\theta=0.15$, the numerical value of $\sigma_{r}$ can exceed $4 \mathrm{kPa}$. If $\theta=0.2$, the value can be $10^{4} \mathrm{~Pa}$. This order of magnitude is already as much as the order of magnitude of the positive pressure stress in the soil-pile interface. And the value of $\sigma_{r}$ will be about $15 \mathrm{kPa}$ if $\theta=0.25$. Note here that the time extreme value of $\sigma_{r}$ appears when $t=2 k \pi / v$ ( $k$ is nonnegative integer), and it is in the equilibrium position (not the situation in which angular displacement reaches its maximum value). This also means that, considering the complexity and randomicity of earthquakes, the actual displacement which is corresponding to the normal stress maximum value in the calculation results can be less than the displacement which was needed in the calculation process. At the same time, although the frequency in most earthquakes is very low (about $2 \sim 10 \mathrm{~Hz}$ ), considering

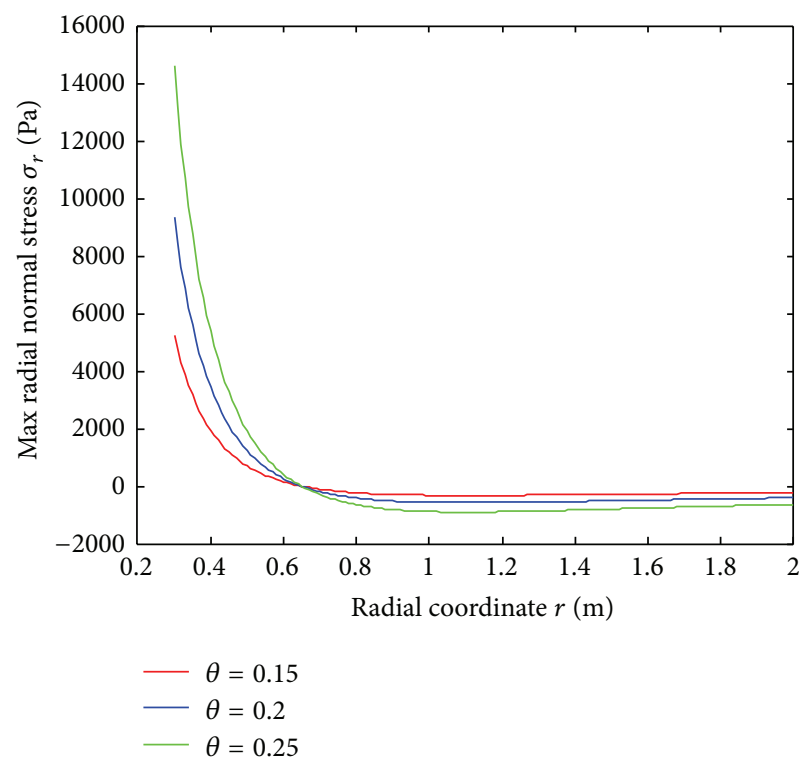

FIGURE 6: Max radial normal stress of soil.

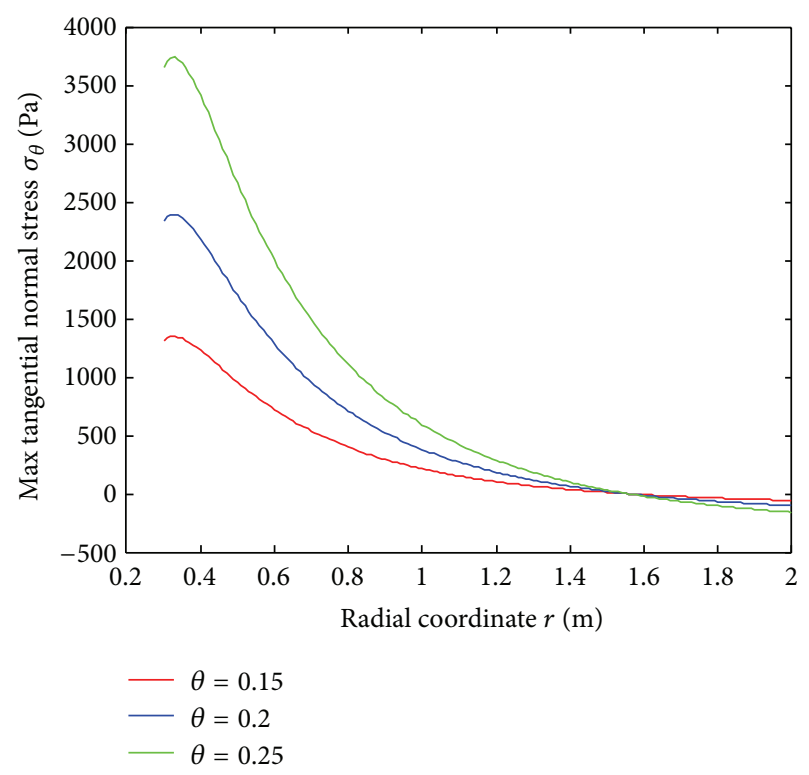

FIGURE 7: Max tangential normal stress of soil.

the complexity and randomicity of earthquake, it is possible that a high frequency appears in a very short time; besides, the frequency used in stress calculation of this section is the instantaneous frequency, not the average frequency of the whole earthquake, and there might be a high frequency in a very short moment during the earthquake which has a low average frequency. At the same time, the rotary movement in this paper is a relative motion of pile and soil. Considering the superposition of the soil's absolute motion, both of the frequency and rotation angle might have a big peak value which will cause a big normal stress in a very short moment, although the absolute motion frequency of the pile is low. So, in an actual earthquake which has a low average frequency 


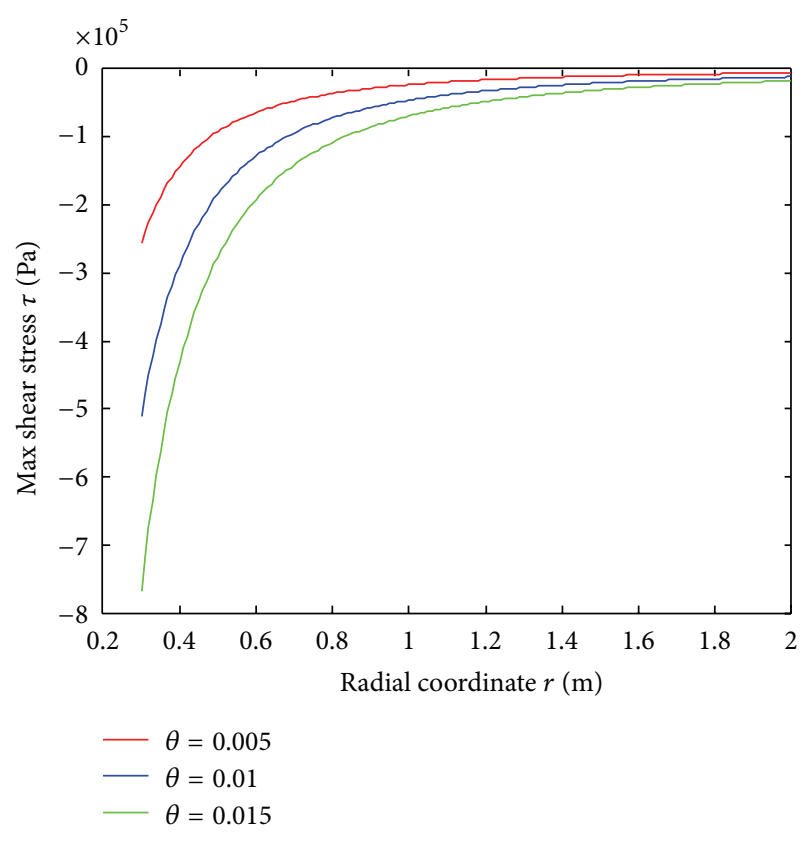

FIGURE 8: Max shear stress of soil.

and a low maximum angular displacement, the order of magnitude of the normal stress could still reach $10^{4} \mathrm{~Pa}$, even higher.

On the other hand, the vertical bearing capacity of rigid friction pile is provided by the vertical friction in the soilpile interface, and the maximum friction force depends on the positive pressure stress in the soil-pile interface. The order of magnitudes of this positive pressure stress is usually $10^{4} \mathrm{~Pa}$ when the depth is about $0 \sim 20 \mathrm{~m}$ to the ground surface; this value is of the same order of magnitude as the positive tensile stress which was calculated in the previous analysis. It means that the tensile stress in the soil-pile interface caused by the centrifugal force will reduce the initial compressive stress in the soil-pile interface during the rotary movement. This will reduce the friction force in the soil-pile interface and then reduce the bearing capacity of the pile.

The shear stress is then analyzed in the following. In fact, the influence of the shear stress is more remarkable. From (41) and (45), it can be seen that the calculation in this section is analyzed approximately as the static problem under the situation that the rotary frequency is low. The direction of the shear stress is horizontal and on the soil-pile interface. The resultant force of the friction force which can be sustained in the soil-pile interface has its upper limit, and this upper limit is decided by the pressure stress in the soil-pile interface. Once the horizontal friction appears, the friction force which can be sustained in the vertical direction will be reduced. So, in the rotary movement, the horizontal shear stress can also reduce the pile's bearing capacity.

Besides, from a numerical point of view, the shear stress is much bigger than the normal stress in the soil-pile interface $(r=0.3 \mathrm{~m})$. Even treating this problem as a static one and without considering the dynamic effect, according to (52), the numerical value of the shear stress in the soil-pile interface is not less than $2 \theta G_{s}$. Considering that the order of magnitude of soil's shear modulus is usually $10^{7} \mathrm{~Pa}$, the shear stress will be more than $20 \mathrm{kPa}$, even if $\theta$ is only $0.1 \%$. In the calculated example, according to Figure 8 , it can be seen that the shear stress will be more than $200 \mathrm{kPa}$, although the rotation angle is only $0.5 \%$. If the rotation angle is $1.5 \%$, the value of the shear stress can be more than $700 \mathrm{kPa}$. When the depth to the ground surface is in the range of $0 \sim 40 \mathrm{~m}$, this shear stress could exceed the shear strength of the soil in the overwhelming majority of cases. That means the soil-pile interface will fail. Not only is the fundamental assumption "the soil-pile interface will not be separated" not valid, but also the mechanism of action in which the friction in the soilpile interface provides the vertical bearing capacity to the pile is false. In this situation, the effect of the soil around the pile is close to lateral loading.

In addition, the following possibility must be considered here: if one pile lost its bearing capacity in a pile group foundation, the loading will be shared by the other piles. Even though the rotary movement ends and the normal stress in the soil-pile interface is recovered, the bearing capacity of this pile is hard to play.

Synthesize all the previous analysis: when the rotary vibration of rigid friction pile occurs, the initial positive pressure stress in the soil-pile interface will be reduced by the dynamic tensile stress which is of the same order of magnitude as the numerical value. That will make the vertical friction force hard to play and reduce the bearing capacity. Besides, the horizontal shear stress does not only weaken the vertical friction force, but also has a very large numerical value even though the rotation angle is very little. That will lead to the failure of the soil-pile interface. Pile will lose its bearing capacity and it is hard to be recovered.

\section{Conclusion and Suggestion}

Based on the previous analysis, this paper gives the following conclusions and suggestions.

Firstly, this paper tried to derive a stress series analytic solution of central symmetry plane elastic problem by using the Laurent expansion method, and three theorems about decoupling, generalized, and convergent properties have been proposed separately. Compared with the integral analytic solution which can only give the point value, this solution can not only give an analytic calculation formula, but also reduce the times of the numerical integration effectively in principle.

Secondly, this paper proposes the concept of "the rotary vibration of rigid friction pile." For the general rotary vibration, the movement of the pile can be seen as rigid rotation when the dimensionless quantity $x=v r / c_{s p}$ is not bigger than 1.84. The displacement of the pile can be given as Dirichlet boundary condition in the calculation of movements of soil around the pile. But the radius of the pile should not be seen as a linear segment if $v r / c_{s p}$ is bigger than 1.84. This may be of some reference value for torsional analysis of piles.

Thirdly, for friction pile foundation, if the earthquake might induce rotary movement of the superstructure, the bearing capacity of the pile of which the rotary movement 
might happen should be taken out in the calculation of the vertical bearing capacity (including the pile which is in the center of the rotary movement at least). For pile group foundation, the influence of this requirement may be not significant. But this influence might be very serious to the foundation of the building such as bridge pier or high-voltage transmission tower of which the number of piles is small.

Finally, for the rotary dynamic problem of rigid pile, it is suggested that the soil around the pile should be taken as lateral loading in the calculation if the soil-pile interface might be a failure.

The above is the conclusion in this paper. In addition, the method in the second section of this paper can also be used in the problem such as variable motion of revolved body or the stress analysis of the well wall. If we use the continuum soil model based on plane strain simplifying assumptions which was presented by Baranov [22], this method can also be applied to the torsional stress analysis of rigid end bearing pile. The details will be introduced in another article.

\section{Conflict of Interests}

The authors declare that there is no conflict of interests regarding the publication of this paper.

\section{Acknowledgments}

The authors sincerely thank the anonymous referees for their valuable suggestions and comments; the work reported in this paper is financially supported by the Self-Initiative Research Program of Tsinghua University (Project no. 2012THZ02-2) and National Key Fundamental Research and Development Program of China (Project no. 2014CB047004).

\section{References}

[1] J. K. Wu, M. Z. Wang, and W. Wang, An Introduction to Mechanics of Elasticity (Revised Edition), Peking University Press, Beijing, China, 2001, (Chinese).

[2] H. G. Poulos, "Torsional response of Piles," Journal of the Geotechnical Engineering Division, vol. 101, no. 10, pp. 1019-1035, 1975.

[3] M. F. Randolph, "Piles subjected to torsion," Journal of the Geotechnical Engineering Division, vol. 107, no. 8, pp. 1095-1111, 1981.

[4] W. D. Guo and M. F. Randolph, "Torsional piles in non-homogeneous media," Computers and Geotechnics, vol. 19, no. 4, pp. 265-287, 1996.

[5] M. Novak and K. Sachs, "Torsional and coupled vibrations of embedded footings," International Journal of Earthquake Engineering and Structural Dynamics, vol. 2, no. 1, pp. 11-33, 1973.

[6] R. K. N. D. Rajapakse and A. H. Shah, "On the lateral harmonic motion of an elastic bar embedded in an elastic half-space," International Journal of Solids and Structures, vol. 23, no. 2, pp. 287-303, 1987.

[7] R. K. N. D. Rajapakse, A. H. Shah, and S. K. Datta, “Torsional vibrations of elastic foundations embedded in an elastic halfspace," Earthquake Engineering and Structural Dynamics, vol. 15, no. 3, pp. 279-297, 1987.

[8] Y. Cai, G. Chen, C. Xu, and D. Wu, "Torsional response of pile embedded in a poroelastic medium," Soil Dynamics and Earthquake Engineering, vol. 26, no. 12, pp. 1143-1148, 2006.
[9] K. Wang, Z. Zhang, C. J. Leo, and K. Xie, "Dynamic torsional response of an end bearing pile in saturated poroelastic medium," Computers and Geotechnics, vol. 35, no. 3, pp. 450-458, 2008.

[10] Z. Q. Zhang, Study on pile torsional vibration in heterogeneous saturated soils [Ph.D. thesis], Zhejiang University, Hangzhou, China, 2008, (Chinese).

[11] J. Boussinesq, Application des Potentiels a l'Equilibre et des Mouvements des Solides Elastiques, Ganthier-Villars, Paris, France, 1885, (French).

[12] H. Neuber, "Ein neuer Ansatz zur Lösung räumlicher Probleme der Elastizitätstheorie. Der Hohlkegel unter Einzellast als Beispiel," Journal of Applied Mathematics and Mechanics, vol. 14, no. 4, pp. 203-212, 1934 (German).

[13] P. M. Naghdi and C. S. Hsu, "On a representation of displacements in linear elasticity in terms of three stress functions," Journal of Applied Mathematics and Mechanics, vol. 10, no. 2, pp. 233-245, 1961.

[14] M. Z. Wang, Advanced Elastic Mechanics, Peking University Press, Beijing, China, 2002, (Chinese).

[15] S. Timoshenko and J. N. Goodier, Theory of Elasticity, McGrawHill, New York, NY, USA, 1951.

[16] L. Kevlin, "Note on the integration of the equations of equilibrium of an elastic solid," in Mathematical and Physical Papers (1), pp. 97-98, Cambridge University Press, Cambridge, UK, 1882.

[17] I. N. Vekua and N. I. Muskhelishvili, "Methods of the theory of analytic functions in the theory of elasticity," in Proceedings of the All-Union Congress on Theoretical and Applied Mechanics (January 27-February 3, 1960), pp. 310-338, Publishing House of the USSR Academy of Sciences, 1962, (Russian).

[18] G. M. Fihtengolts, Course of Differential and Integral Calculus, vol. 2, State Publishing House of Physical and Mathematical Literature, Moscow, Russia, 4th edition, 1959, (Russian).

[19] E. C. Young, Vector and Tensor Analysis, Marcel Dekker, New York, NY, USA, 1978.

[20] Z. H. Ni, Mechanics of Vibration, Xi'an Jiaotong University Press, Xian, China, 1989, (Chinese).

[21] Z. X. Wang and D. R. Guo, Special Functions, World Scientific Press, Singapore, 1989.

[22] V. A. Baranov, "On the calculation of excited vibrations of an embedded foundation," Voprosy Dynamiki Prochnocti, Polytechnic Institute of Riga, pp. 195-209, 1967 (Russian). 


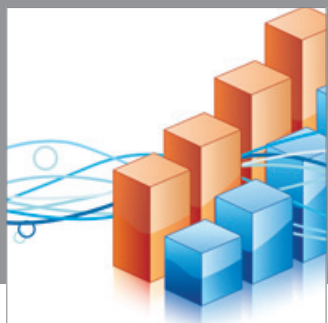

Advances in

Operations Research

mansans

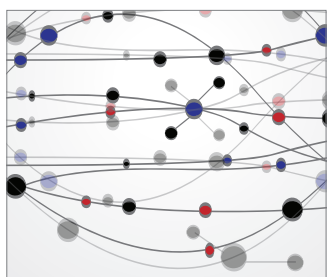

The Scientific World Journal
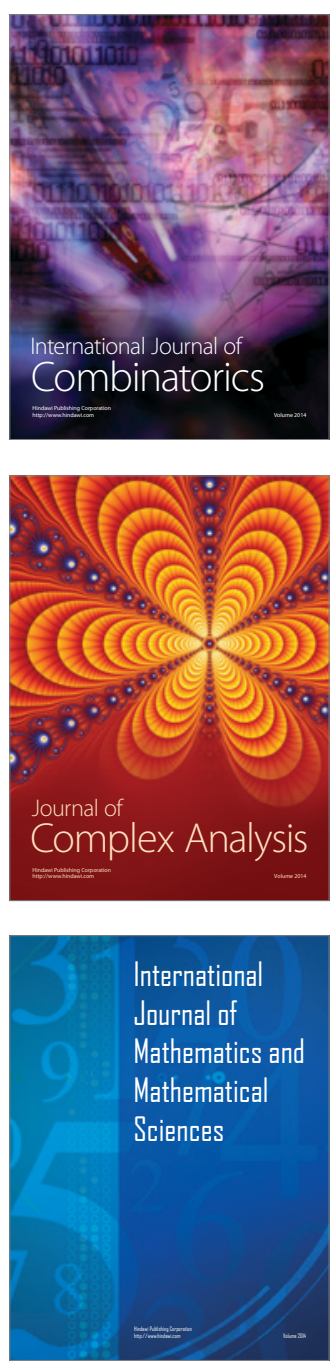
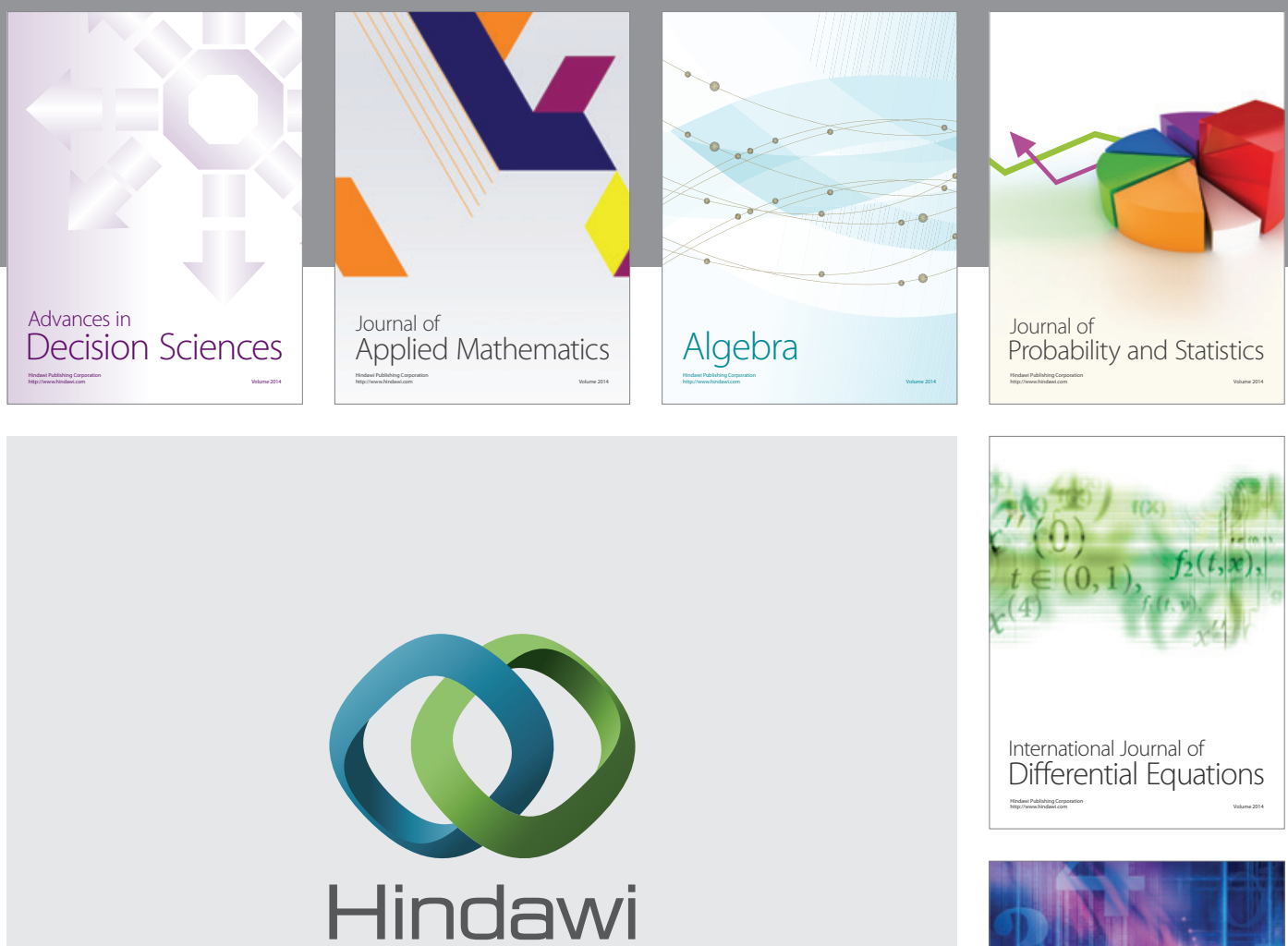

Submit your manuscripts at http://www.hindawi.com
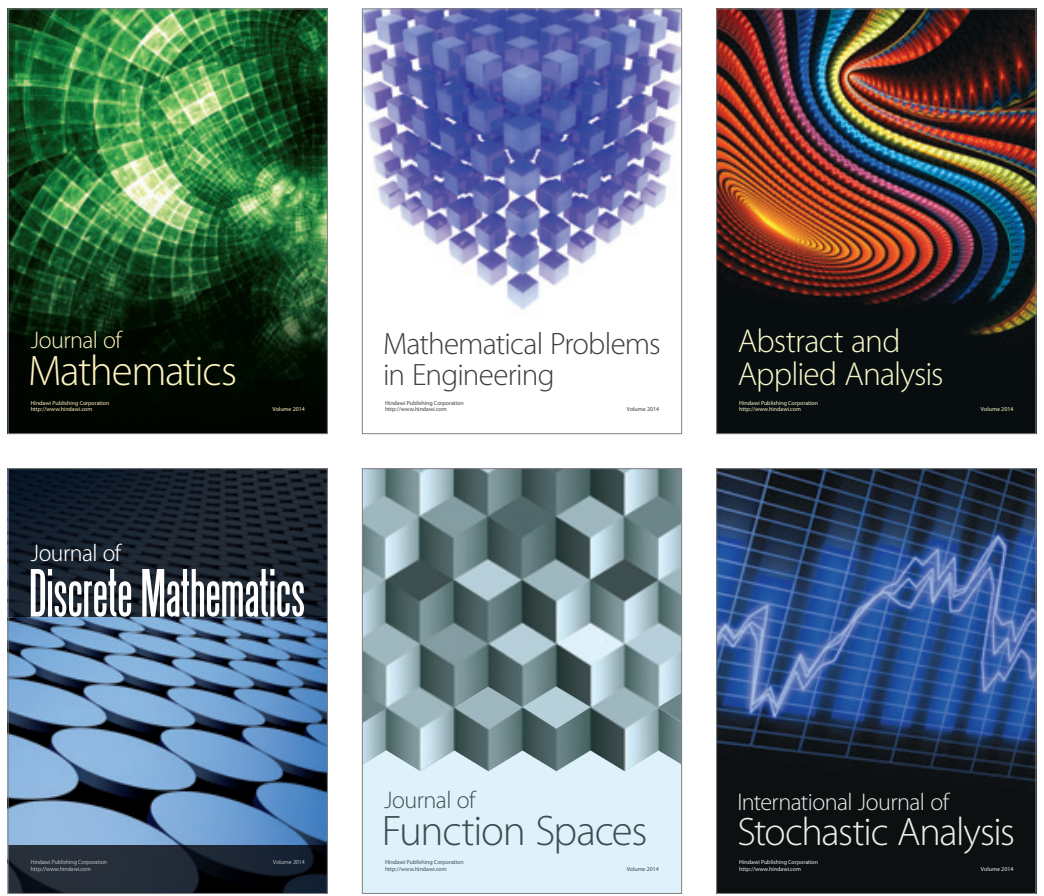

Journal of

Function Spaces

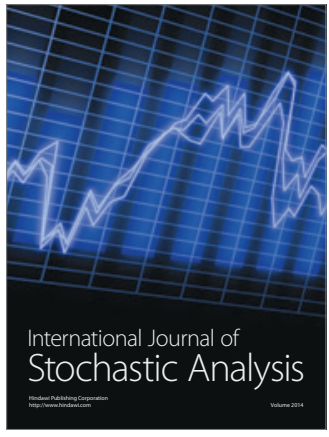

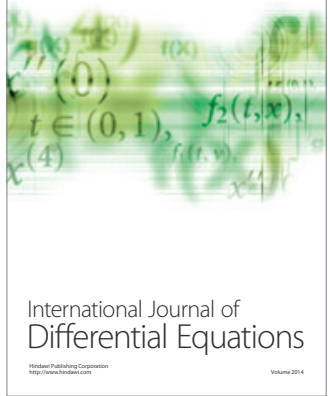
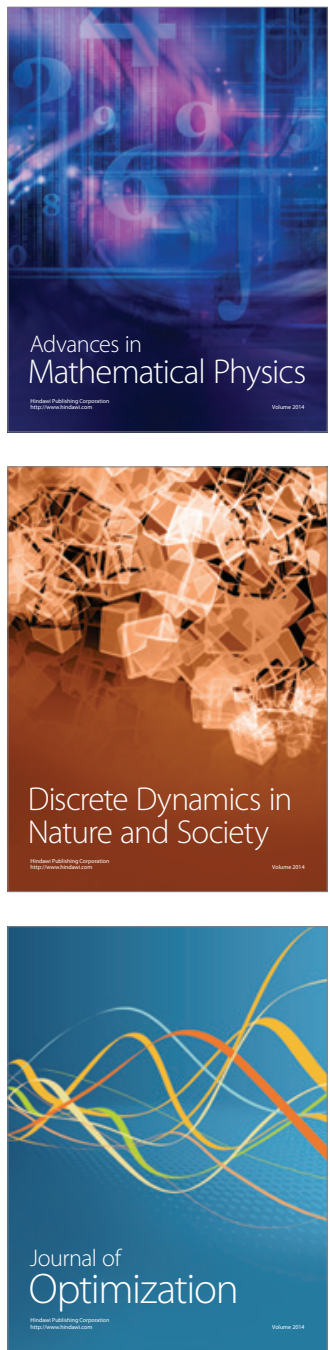